\title{
Instruments and equipment monitoring system based on the internet of things technology
}

\author{
Meng Su, a , Lianghe $\mathrm{Su}^{2, \mathrm{~b}}$ and Hongjiang $\mathrm{Hu}^{3, \mathrm{c}}$ \\ ${ }^{1}$ China CEPREI Laboratory, Dongguanzhuang Rd, Tianhe area ,Guangzhou, China \\ ${ }^{2}$ China CEPREI Laboratory, Dongguanzhuang Rd, Tianhe area ,Guangzhou,China \\ ${ }^{3}$ China CEPREI Laboratory, Dongguanzhuang Rd, Tianhe area ,Guangzhou,China \\ asum@ceprei.biz, ${ }^{\mathrm{a}}$ slh@ceprei.biz, ${ }^{\mathrm{c}} \mathrm{hhj} @$ ceprei.biz
}

\begin{abstract}
Keywords: Instruments and Equipment Monitoring System; Automated Testing; LabVIEW Platform Abstract. In this paper, to the equipment monitoring as the research target, we analyzed the usage demand of automatic monitoring instruments and established equipment of automatic monitoring system based on the Internet of things technology. System adopts open and modular design of frame structure and flexible hardware, can according to customer requirements of product testing hardware configuration, has high cost performance and good generality. Real time display automatic test process, test results, and can be carried out on the test data storage, statistical analysis and report printing, etc.
\end{abstract}

\section{Introduction}

For daily use process of monitoring instruments, improve the efficiency of using equipment, this will have especially important significance to establish equipment running condition monitoring system based on Internet of things. This study will focus on different instruments and equipment in the course of everyday use switch machine, working time, power consumption and status of remote monitoring, transmission, recording and analysis of management, easy to master by the instrument use and management of each instrument for a period of time[1].

Instruments and equipment for daily use monitoring system to realize the instruments and equipment in different departments of the remote wireless or LAN monitoring, the server software for real-time analysis of testing data, it is concluded that the instrument for a period of time of use[2].

\section{The establishment of the hardware model}

The establishment of the model will give full consideration to the existing mature technology, follow the principle of practicality, stability, security and reliability. According to different using requirements, this paper will establish a remote wireless and wired two functions: wireless solution refers to the use of existing mature GPRS network or TDMA network, realize the data remote wireless transmission; Local area network (LAN) cable plan refers to the use of the existing foundation, instruments and equipment parameters through LAN to the server to achieve unified management. Specific implementation process is as follows:

First build complete hardware framework, including the accurate current detection module, RFID radio frequency identification tag, a serial port RS232/485 and GPRS network conversion module or network interface, sending module, remote server receiving module and the server[3]. Test module will real-time accurate testing instrument of current, an RFID label for each device, real-time to record the location of each device and equipment basic information such as attribute, and the current value and equipment information, location, etc. Through the GPRS network or local area network (LAN), using the TCP/UDP/IP protocol wireless remote sent to the server, the server monitoring software is judged the current work status and recorded through the analysis of the current value of different instruments, in order to realize the various instruments and equipment for a period of time of the switch machine, working time and power consumption. System hardware block diagram is shown in Fig. 1: 


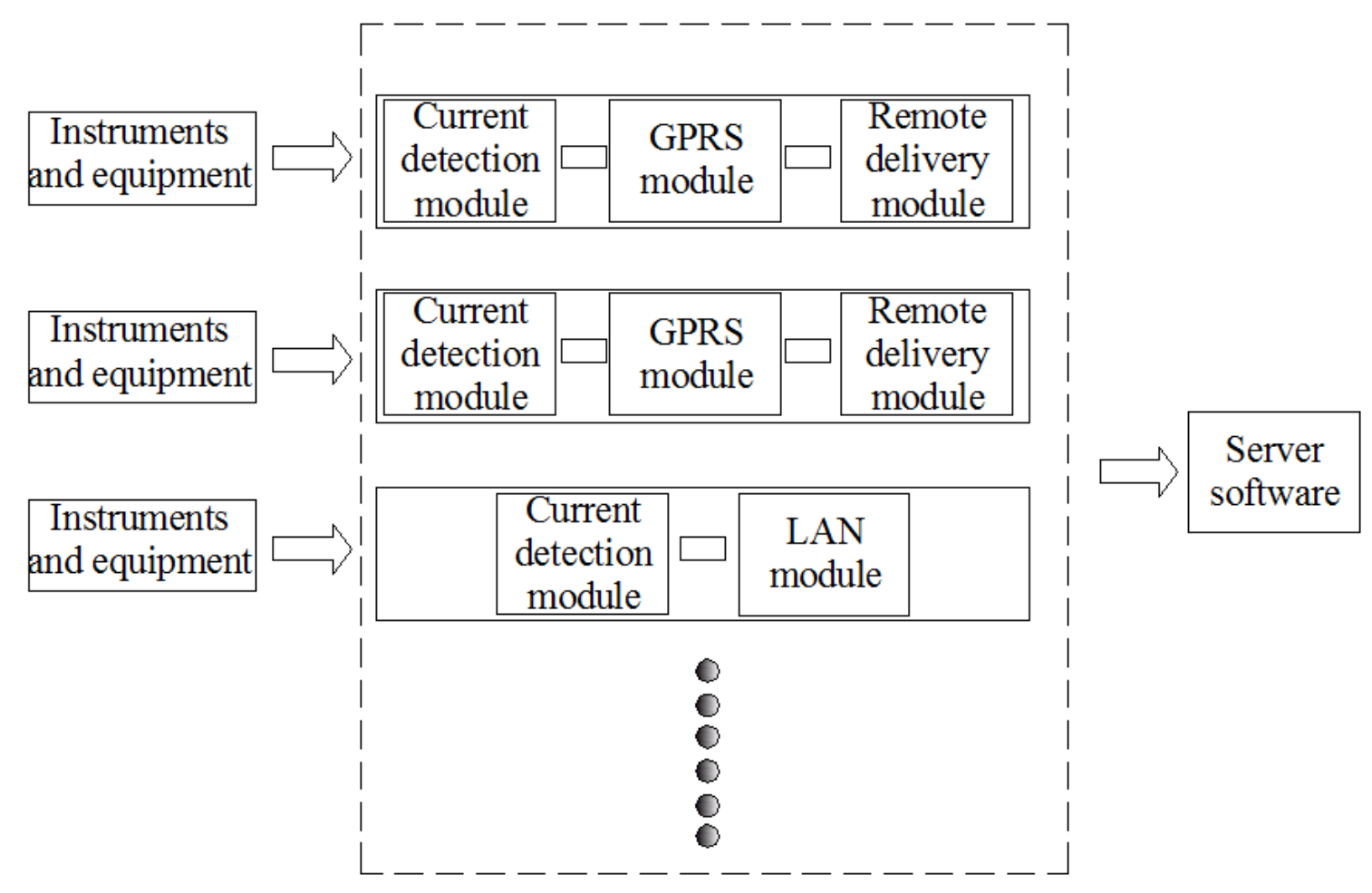

Fig.1. hardware block diagram of the system

\section{Software model analysis and function implementation}

First of all need to consider the selection of design platform designed in this paper using LabVIEW as the software design. LabVIEW (Laboratory Virtual instrument Engineering) is a graphical programming language, it is widely accepted by the industry, academia and research Laboratory, as a standard data acquisition and instrument control software. In LabVIEW VISA module can control GPIB, serial, USB, Ethernet, PXI or VXI instrument, and according to the type of instrumentation to invoke the corresponding driver, users need to learn all kinds of instrument communication protocol. VISA is independent of the operating system, bus and the programming environment. In other words, no matter use what kind of equipment, operating system and programming language, both use the same API. Compared with the other programming language, such as LabVIEW integrated modules can greatly shorten the development cycle, the interface is beautiful and easy, reconfigurable[4].

System mainly make use of the communication interface, such as 485 , according to the communication protocol to complete the collection of parameters. LabSQL toolkit was adopted to access database and realize real-time data storage, combining the theory of probability and statistics and so on product quality management, using LabVIEW statistics module, realize the bad project analysis, process capability index, measuring control chart and other statistical analysis function.

This system design and development under the environment of LabVIEW software, use a modular, hierarchical design method according to the general requirements and performance parameters of system, the software is divided into several different functional modules and three layers to the VI of the basic implementation. In high-level application users can implement test plan edition, execute the test and data analysis, etc; Middle function is the implementation of command interpretation, test process control, instrument control and data read, algorithm, analysis test data, and other functions; The underlying drivers including LabVIEW development environment, instrument driver interface and database driver. A key part of the system is to perform the test.

Test function according to system requirements, the monitoring software based on LabVIEW platform is versatile, realize remote automatic monitoring and analysis of the various instruments, specific test function is as follows: automatic real-time monitoring; test information, status, parameter 
real-time display; Automatic storage test data; Test result automatic judgment and analysis; Hardware can be expanded and replacement[5].

After finishing the development of hardware and software debugging, the system will implement the remote automatic monitoring and analysis of the various instruments, specific function effect is as follows: the number of instruments and equipment monitoring can be extended; Automatic transmission system through the wireless network, there is no limit to the distance; Each instrument equipment working condition can be in online real-time monitoring software (whether in work or standby); Each instrument can monitor information, the parameters (instrument location, name, instrument working current, etc.); All the monitoring data of automatic storage; Each instrument can inquire on the software equipment usage for a period of time; Each instrument can inquire the power consumption over a period of time.

As shown in Fig. 2, the system software design simple, friendly interface, suitable for test engineer, with automatic testing, system integration, automatic data collection, data storage, data processing and analysis, report generation, etc.

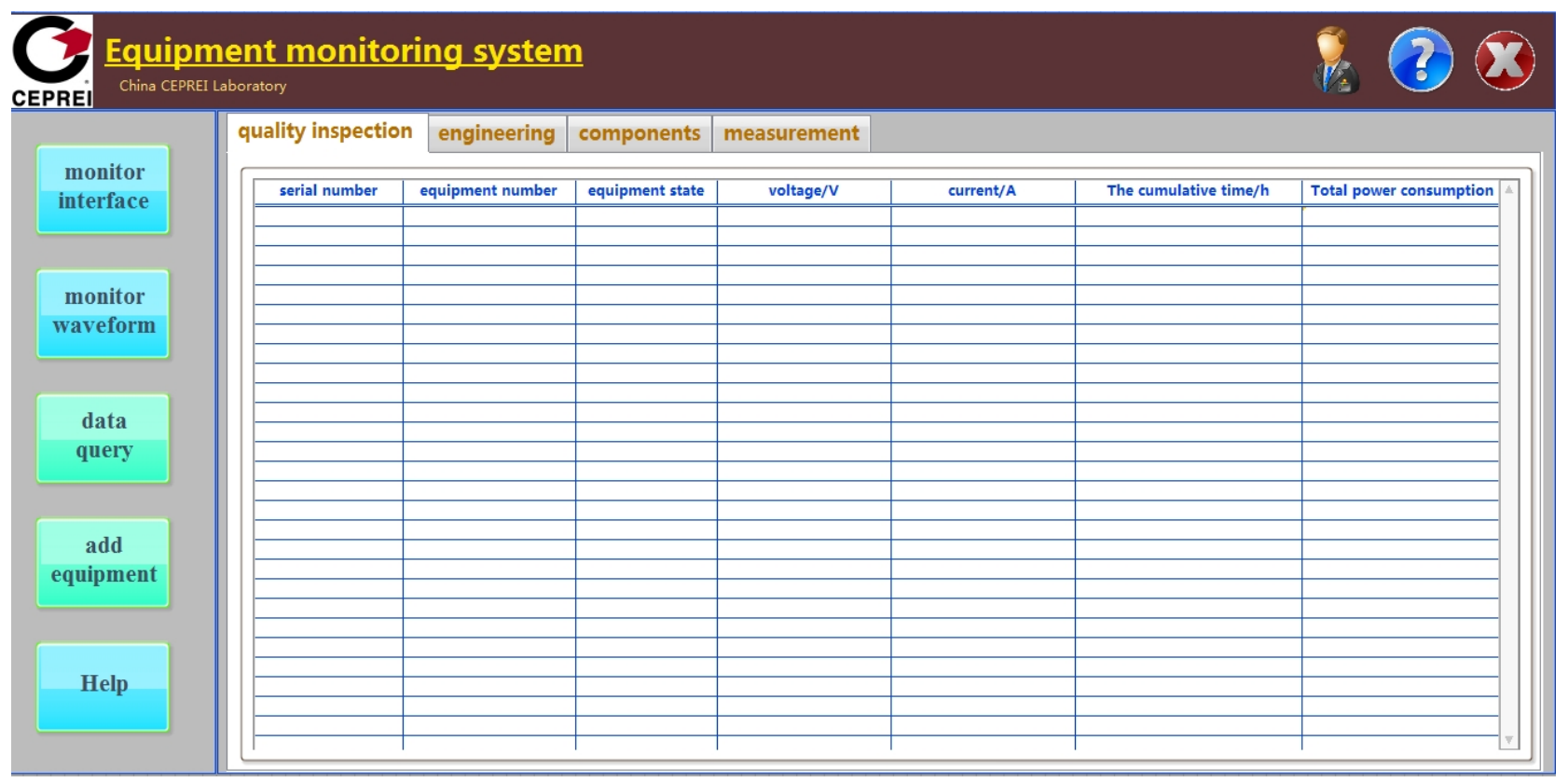

Fig.2. software interface diagram

\section{The main technical difficulties and solutions of the system}

How to realize the stable over a long distance monitoring data transmission is core difficult problem in the project. This scheme will through GPRS, TDMA network or local area network (LAN), using the TCP/UDP/IP protocol to send test data to a server, the server monitoring software based on the analysis of different address to upload data and document analysis, the advantage is the use of mature network, is not restricted by distance, realize the stability of the signal data transmission.

The stability of a large number of instruments and equipment monitoring. This scheme by adopting mature telecommunications network or local area network (LAN), at the same time solve the distance facing common bus or quantitative restrictions, can be arbitrarily extended to the whole system.

System need to monitor the working condition of all the instruments for a long time, the large amount of data storage, real-time multitasking operating and processing, will be to consider the problems in the process of software development. This solution will monitor software based on LabVIEW development platform is versatile, and use the built-in database module development corresponding database software, the software adopts modular programming method, easy to maintenance and upgrades in the late. Considering the stable operation of the system, will use a high performance server as a system of PC, all data storage and analysis, enough to cope with the stable operation of the system. 


\section{Conclusions}

For example, based on equipment monitoring, automatic monitoring instruments and equipment usage demand is analyzed, build equipment of automatic monitoring system based on the Internet of things technology.

System adopts open and modular design of frame structure and flexible hardware, can according to customer requirements of product testing hardware configuration, has high cost performance and good generality. Real time display automatic test process, test results, and can be carried out on the test data storage, statistical analysis and report printing, etc.

Through the establishment of this system has realized the automation of the whole experiment equipment usage monitoring, greatly saves the time of the equipment management engineer and reduce labor costs, and improve the automation degree of equipment management and data reliability.

\section{References}

[1] Yun Xu. Design automated test system of the next generation [J]. Today's electronic ,2008,8:58-61

[2] Xiangru Shan. software will be the core of the next generation automation system [J]. Electronic design technology, 2008,7:122

[3] What's the NI Teststandard. National Instruments , 2008

[4] Yingbin Dong, Bing Han. The interface research of using ActiveX technology access to Excel in LabVIEW [J]. Micro computer information. 2006,(04):165-166.

[5] Leping Yang, Haitao Li. LabVIEW high-level programming [M]. Beijing: TsingHua university press. 